\title{
EXTENSIONS OF VALUATIONS TO THE HENSELIZATION AND COMPLETION
}

\author{
STEVEN DALE CUTKOSKY
}

\section{INTRODUCTION}

Suppose that $K$ is a field. Associated to a valuation $\nu$ of $K$ is a value group $\Phi_{\nu}$ and a valuation $\operatorname{ring} V_{\nu}$ with maximal ideal $m_{\nu}$. Let $R$ be a local domain with quotient field $K$. We say that $\nu$ dominates $R$ if $R \subset V_{\nu}$ and $m_{\nu} \cap R=m_{R}$ where $m_{R}$ is the maximal ideal of $R$. We have an associated semigroup

$$
S^{R}(\nu)=\{\nu(f) \mid f \in R \backslash(0)\},
$$

as well as the associated graded ring of $R$ along $\nu$

$$
\operatorname{gr}_{\nu}(R)=\bigoplus_{\gamma \in \Phi_{\nu}} \mathcal{P}_{\gamma}(R) / \mathcal{P}_{\gamma}^{+}(R)=\bigoplus_{\gamma \in S^{R}(\nu)} \mathcal{P}_{\gamma}(R) / \mathcal{P}_{\gamma}^{+}(R)
$$

which is defined by Teissier in [18]. Here

$$
\mathcal{P}_{\gamma}(R)=\{f \in R \mid \nu(f) \geq \gamma\} \text { and } \mathcal{P}_{\gamma}^{+}(R)=\{f \in R \mid \nu(f)>\gamma\} .
$$

This ring plays an important role in local uniformization of singularities ([18] and [19]). The ring $\operatorname{gr}_{\nu}(R)$ is a domain, but it is often not Noetherian, even when $R$ is. In fact, a necessary condition for $\operatorname{gr}_{\nu}(R)$ to be Noetherian is that $\Phi_{\nu}$ be a finitely generated group.

In this paper, we answer the following question, which is a natural generalization of local uniformization.

Question 1.1. Suppose that $R$ is a Noetherian local domain which is dominated by a valuation $\nu$. Does there exist a regular local ring $R^{\prime}$ of the quotient field $K$ of $R$ such that $\nu$ dominates $R^{\prime}$ and $R^{\prime}$ dominates $R$, a prime ideal $p$ of the $m_{R^{\prime}}$-adic completion $\widehat{R^{\prime}}$ of $R^{\prime}$ such that $p^{\prime} \cap R^{\prime}=(0)$ and an extension $\hat{\nu}$ of $\nu$ to the quotient field of $\widehat{R^{\prime}} / p$ which dominates $\widehat{R^{\prime}} / p$ such that

$$
\operatorname{gr}_{\nu}\left(R^{\prime}\right) \cong \operatorname{gr}_{\hat{\nu}}\left(\widehat{R^{\prime}} / p\right) ?
$$

A nonzero prime ideal $p$ may be necessary to obtain the conclusions of Question 1.1, as is shown in [17] and [10.

If $\nu$ has rank 1 , then we easily obtain a prime $p$ in the completion of $R$ such that

$$
\operatorname{gr}_{\nu}(R) \cong \operatorname{gr}_{\hat{\nu}}(\hat{R} / p)
$$

as we now indicate.

Suppose that $R$ is a Noetherian local domain which is dominated by a rank 1 valuation $\nu$. For $f \in \hat{R}$, we write $\nu(f)=\infty$ if there exists a Cauchy sequence $\left\{f_{n}\right\}$ in $R$ which converges to $f$, and such that $\lim _{n \rightarrow \infty} \nu\left(f_{n}\right)=\infty$. We define ([7, Definition 5.2]) a prime ideal

$$
P(\hat{R})_{\infty}=\{f \in \hat{R} \mid \nu(f)=\infty\}
$$

partially supported by NSF grant DMS-1700046. 
in $\hat{R}$. We then have a canonical immediate extension $\hat{\nu}$ of $\nu$ to $\mathrm{QF}\left(\hat{R} / P(\hat{R})_{\infty}\right)$ which dominates $\hat{R} / P(\hat{R})_{\infty}$.

The following lemma appears in [11].

Lemma 1.2. Suppose that $\nu$ is a rank 1 valuation of a field $K$ and $R$ is a Noetherian local domain which is dominated by $\nu$. Let $\hat{\nu}$ be the canonical extension of $\nu$ to $\operatorname{QF}\left(\hat{R} / P(\hat{R})_{\infty}\right)$ which dominates $\hat{R} / P(\hat{R})_{\infty}$. Then the inclusion $R \rightarrow \hat{R} / P(\hat{R})_{\infty}$ induces an isomorphism

$$
\operatorname{gr}_{\nu}(R) \cong \operatorname{gr}_{\hat{\nu}}\left(\hat{R} / P(\hat{R})_{\infty}\right)
$$

Proof. Suppose $h \in \hat{R} \backslash P(\hat{R})_{\infty}$. There exists a Cauchy sequence $\left\{f_{n}\right\}$ in $R$ such that $\lim _{n \rightarrow \infty} f_{n}=h$. Let $m$ be a positive integer such that $m \nu\left(m_{R}\right)>\hat{\nu}(h)$ (where $\nu\left(m_{R}\right)=$ $\left.\min \left\{\nu(g) \mid g \in m_{R}\right\}\right)$. There exists $n_{0}$ such that $f_{n}-h \in m_{R}^{m} \hat{R}$ for $n \geq n_{0}$. Then $\operatorname{in}_{\nu}\left(f_{n}\right)=\operatorname{in}_{\hat{\nu}}(h)$ for $n \geq n_{0}$.

From Lemma 1.2, we obtain a positive answer to Question 1.1 for local domains $R$ and rank 1 valuations $\nu$ which admit local unformization. A positive answer to Question 1.1 for rank 1 valuations with the additional conclusion that $\widehat{R^{\prime}} / p$ is a regular local ring is given in [4] and in [7, Theorem 7.2] for $R$ which are essentially of finite type over a field of characteristic zero. This is generalized somewhat in [6] and [9].

Related to Question 1.1 is the following question, which we will also answer.

Question 1.3. Suppose that $R$ is a Noetherian local domain which is dominated by a valuation $\nu$. Does there exist a regular local ring $R^{\prime}$ of the quotient field $K$ of $R$ such that $\nu$ dominates $R^{\prime}$ and $R^{\prime}$ dominates $R$, and an extension $\nu^{h}$ of $\nu$ to the quotient field of the Henselization $\left(R^{\prime}\right)^{h}$ of $R^{\prime}$ which dominates $\left(R^{\prime}\right)^{h}$ such that

$$
\left.\operatorname{gr}_{\nu}\left(R^{\prime}\right) \cong \operatorname{gr}_{\nu^{h}}\left(\left(R^{\prime}\right)^{h}\right)\right) ?
$$

A positive answer to Question 1.1 would imply a positive answer to Question 1.3 .

We prove the following proposition on the extension of associated graded rings under an unramified extension in [5], which gives a start on answering Question 1.3. Related problems are considered in [11].

Proposition 1.4. ([5, Proposition 1.7]) Suppose that $R$ and $S$ are normal local rings such that $R$ is excellent, $S$ lies over $R$ and $S$ is unramified over $R, \tilde{\nu}$ is a valuation of the quotient field $L$ of $S$ which dominates $S$, and $\nu$ is the restriction of $\tilde{\nu}$ to the quotient field $K$ of $R$. Suppose that $L$ is finite over $K$. Then there exists a normal local ring $R^{\prime}$ of $K$ which is dominated by $\nu$ and dominates $R$, such that if $R^{\prime \prime}$ is a normal local ring of $K$ which is dominated by $\nu$ and dominates $R^{\prime}$ and $S^{\prime \prime}$ is the normal local ring of $L$ which is dominated by $\tilde{\nu}$ and lies over $R^{\prime \prime}$, then $R^{\prime \prime} \rightarrow S^{\prime \prime}$ is unramified, and

$$
\operatorname{gr}_{\tilde{\nu}}\left(S^{\prime \prime}\right) \cong \operatorname{gr}_{\nu}\left(R^{\prime \prime}\right) \otimes_{R^{\prime \prime}} / m_{R^{\prime \prime}} S^{\prime \prime} / m_{S^{\prime \prime}}
$$

We give an example at the end of of [5, Section 5] showing that it may be necessary to take $R^{\prime} \neq R$ to obtain the conclusions of Proposition 1.4 if $\nu$ has rank greater than 1. The ring $R$ is regular and there is no residue field extension in the example. This example shows that it may be necessary to perform a proper extension $R \rightarrow R^{\prime}$ take $R^{\prime} \neq R$ to obtain a positive answer to Question [1.3 or 1.1] even if $R$ is a regular local ring. This problem arises from the fact that the residue field under blowing up of the center of a composite valuation can increase. Related examples are considered in [11]. In [11, Remark 2], it is already observed that the increase of residue field under blowing up of 
the center of a composite valuation is a critical issue in understanding an extension of a valuation dominating a local domain to its completion.

In this paper, we show that Question 1.3 and Question 1.1 have a negative answer in general. This is accomplished in Theorems 1.5 and 1.6 stated below, which are proven in Section 5. The examples of the theorems are on three dimensional regular local rings which are a localization at a maximal ideal of a polynomial ring over an arbitrary algebraically closed field.

Theorem 1.5. Suppose $k$ is an algebraically closed field. Then there exists a three dimensional regular local ring $T_{0}$, which is a localization of a finite type $k$-algebra, with residue field $k$, and a valuation $\varphi$ of the quotient field $K$ of $T_{0}$ which dominates $T_{0}$ and whose residue field is $k$, such that if $T$ is a regular local ring of $K$ which is dominated by $\varphi$ and dominates $T_{0}, T^{h}$ is the Henselization of $T$ and $\varphi^{h}$ is an extension of $\varphi$ to the quotient field of $T^{h}$ which dominates $T^{h}$, then $S^{T^{h}}\left(\varphi^{h}\right) \neq S^{T}(\varphi)$, under the natural inclusion $S^{T}(\varphi) \subset S^{T^{h}}\left(\varphi^{h}\right)$.

Theorem 1.6. Suppose $k$ is an algebraically closed field. Then there exists a three dimensional regular local ring $T_{0}$, which is a localization of a finite type $k$-algebra, with residue field $k$, and a valuation $\varphi$ of the quotient field $K$ of $T_{0}$ which dominates $T_{0}$ and whose residue field is $k$, such that if $T$ is a regular local ring of $K$ which is dominated by $\varphi$ and dominates $T_{0}$ and $\hat{T}$ is the $m_{T}$-adic completion of $T$, then there does not exist a prime ideal $p$ of $\hat{T}$ such that $p \cap T=(0)$ with an extension $\hat{\varphi}$ to the quotient field of $\hat{T} / p$ which dominates $\hat{T} / p$ such that $S^{\hat{T} / p}(\hat{\varphi})=S^{T}(\varphi)$, under the natural inclusion $S^{T}(\varphi) \subset S^{\hat{T} / p}(\hat{\varphi})$.

A very interesting related problem, which is still open, is [11, Conjecture 1.1] on the existence of "scalewise birational" extensions of associated graded rings. [11, Conjecture 1.1] is a refinement of [18, Statement 5.19]. The two results Theorem 1.5] and Theorem [1.6] of this paper are counterexamples to possible hopes of improving the statements of Conjecture 1.11 and Theorem 7.1 of [11].

This paper relies on the construction of generating sequences (Section 3), using the algorithm of [8], which is a generalization of the algorithm of [17]. The construction of generating sequences in a local domain which is dominated by a valuation which provide enough information to determine the associated graded ring along the valuation is an important problem. Some recent papers addressing this are [9], [12], [14] and [16].

\section{Notation}

The nonnegative integers will be denoted by $\mathbb{N}$ and the positive integers will be denoted by $\mathbb{Z}_{+}$. If $\Lambda$ is a subset of an Abelian group $G$ then $G(\Lambda)$ will denote the group generated by $\Lambda$ and $S(\Lambda)$ will denote the semigroup (containing zero) generated by $\Lambda$.

The maximal ideal of a local ring $R$ will be denoted by $m_{R}$. Suppose that $K$ is a field. A local ring of $K$ is a local domain whose quotient field is $K$. We will say that a local domain $B$ dominates a local domain $A$ if $A \subset B$ and $m_{B} \cap A=m_{A}$. If a regular local ring $B$ dominates a local domain $A$ and $B$ is a local ring of the blow up of an ideal $I$ in $A$, then a strict transform of an element $f \in A$ in $B$ is a generator $g$ of the principal ideal $(f):_{B} I B$; that is, $g$ is a generator of the ideal of the strict transform of $\operatorname{Spec}(A /(f))$ in $\operatorname{Spec}(B)$.

If $\nu$ is a valuation of a field $K, V_{\nu}$ will denote the valuation ring of $\nu, m_{\nu}$ will denote the maximal ideal of $V_{\nu}$ and $\Phi_{\nu}$ will denote the value group of $\nu$. The basics of valuation 
theory are explained in [21, Chapter VI] and [3, Chapter II]. We will say that a valuation $\nu$ dominates a local domain $R$ if $V_{\nu}$ dominates $R$. We define the semigroup

$$
S^{R}(\nu)=\{\nu(f) \mid f \in R \backslash\{0\}\} .
$$

\section{Construction of generating sequences of a valuation}

Suppose that $R$ is a local domain and $\nu$ is a valuation dominating $R$. A sequence of elements $\left\{P_{i}\right\}$ in $R$ is called a generating sequence for $\nu$ if the classes $\operatorname{in}_{\nu}\left(P_{i}\right)$ of the $P_{i}$ in $\mathcal{P}_{\nu\left(P_{i}\right)} / \mathcal{P}_{\nu\left(P_{i}\right)}^{+}$generate $\operatorname{gr}_{\nu}(R)$ as an $R / m_{R^{-}}$-algebra.

Let $k$ be an algebraically closed field, The regular local ring $R_{0}=k[x, y, t]_{(x, y)} \cong$ $k(t)[x, y]_{(x, y)}$ has regular parameters $x, y$ and residue field $R_{0} / m_{R_{0}} \cong k(t)$. We will inductively define a generating sequence

$$
P_{0}=x, P_{1}=y, P_{2}, \ldots
$$

in $R_{0}$, defining a valuation $\nu$ dominating $R_{0}$, using the method of the proof of $[8$, Theorem 1.1]. The generating sequence will satisfy the good conditions of the conclusions of 8 , Theorem 4.2]. Let $\bar{p}_{1}, \bar{p}_{2}, \ldots$ be the sequence of prime numbers, excluding the characteristic of $k$. Define $a_{1}=\bar{p}_{1}+1$ and inductively define positive integers $a_{i}$ by

$$
a_{i+1}=\bar{p}_{i} \bar{p}_{i+1} a_{i}+1 \text {. }
$$

Define

$$
P_{i+1}=P_{i}^{\bar{p}_{i}^{2}}-(1+t) x^{\bar{p}_{i} a_{i}}
$$

for $i \geq 1$. Set $\nu(x)=1$ and

$$
\nu\left(P_{i}\right)=\frac{a_{i}}{\bar{p}_{i}} \nu(x) \text { for } i \geq 1 .
$$

We have $\operatorname{gcd}\left(a_{i}, \bar{p}_{i}\right)=1$ for all $i$. Thus $G\left(\nu\left(P_{0}\right), \nu\left(P_{1}\right)\right)=\frac{1}{\bar{p}_{1}} \mathbb{Z}$ and

$$
\bar{n}_{i}:=\left[G\left(\nu\left(P_{0}\right), \ldots, \nu\left(P_{i}\right)\right): G\left(\nu\left(P_{0}\right), \ldots, \nu\left(P_{i-1}\right)\right]=\bar{p}_{i}\right.
$$

for $i \geq 1$.

We have $\nu\left(P_{i}\right)>\bar{p}_{i-1}^{2} \nu\left(P_{i-1}\right)$ for $i \geq 2$ and the value group

$$
\Phi_{\nu}=\cup_{i \geq 1} \frac{1}{\bar{p}_{1} \bar{p}_{2} \cdots \bar{p}_{i}} \mathbb{Z}
$$

Let $\varkappa$ be an algebraic closure of $k(t)$ and let $\alpha_{i} \in \varkappa$ be a root of $f_{i}(u)=u^{\bar{p}_{i}}-(1+t) \in k[u]$ for $i \geq 1$. Since the $\bar{p}_{i}$ are all pairwise relatively prime, by induction on $i$, we see that $f_{i}(u)$ is the minimal polynomial of $\alpha_{i}$ over $k\left(\alpha_{1}, \ldots, \alpha_{i-1}\right)$ and

$$
d_{i}:=\bar{p}_{i}=\left[k(t)\left(\alpha_{1}, \ldots, \alpha_{i}\right): k(t)\left(\alpha_{1}, \ldots, \alpha_{i-1}\right)\right]
$$

for $i \geq 1$. Since $n_{i}=d_{i} \bar{n}_{i}=\bar{p}_{i}^{2}$ for $i \geq 1$, by the algorithm of the proof of [8, Theorem 1.1], the $P_{i}$ are the generating sequence of a valuation $\nu$ dominating $R_{0}$ which has the property that setting $U_{i}=x^{a_{i}}$ for $i \geq 1$, we have $V_{\nu} / m_{\nu}=k\left(\left\{\alpha_{i} \mid i \geq 1\right\}\right)$ with

$$
\alpha_{i}=\left[\frac{P_{i}^{\bar{p}_{i}}}{U_{i}}\right] \in V_{\nu} / m_{\nu}
$$

for $i \geq 1$.

From $\bar{p}_{1} \nu(y)=a_{1} \nu(x)$ with $\operatorname{gcd}\left(a_{1}, \bar{p}_{1}\right)=1$, there exist $\bar{a}_{0}, \bar{b}_{0} \in \mathbb{Z}_{+}$such that $\bar{p}_{1} \bar{b}_{0}-$ $a_{1} \bar{a}_{0}=1$. Set

$$
x=x_{1}^{\bar{p}_{1}} \tilde{y}_{1}^{\bar{a}_{0}}, y=x_{1}^{a_{1}} \tilde{y}_{1}^{\bar{b}_{0}} .
$$


Then $\nu\left(x_{1}\right)>0$ and $\nu\left(\tilde{y}_{1}\right)=0$. Set

$$
R_{1}=R_{0}\left[x_{1}, \tilde{y}_{1}\right]_{m_{\nu} \cap R_{0}\left[x_{1}, \tilde{y}_{1}\right]} .
$$

By [8, Theorem 7.1],

$$
x_{1}, y_{1}=\frac{P_{2}}{x_{1}^{a_{1} \bar{p}_{1}^{2}}}
$$

are regular parameters in $R_{1}$ and

$$
\begin{gathered}
P_{0,1}=x_{1}, P_{1,1}=\frac{P_{2}}{P_{0,1}^{a_{1} \bar{p}_{1}^{2}}}, \\
P_{i, 1}=\frac{P_{i+1}}{P_{0,1}^{a_{1} \bar{p}_{1}^{2} \cdots \bar{p}_{i}^{2}}}
\end{gathered}
$$

for $i \geq 1$ is a generating sequence for $\nu$ in $R_{1}$. For $i \geq 1$, we have

$$
P_{i+1,1}=\frac{P_{i+2}}{P_{0,1}^{a_{1} \bar{p}_{1}^{2} \cdots \bar{p}_{i+1}^{2}}}=P_{i, 1}^{\bar{p}_{i+1}^{2}}-(1+t) \tilde{y}_{1}^{\bar{a}_{0} a_{i+1} \bar{p}_{i+1}} x_{1}^{\bar{p}_{i+1}\left(\bar{p}_{1} a_{i+1}-a_{1} \bar{p}_{1}^{2} \cdots \bar{p}_{i}^{2} \bar{p}_{i+1}\right)} .
$$

Set

and

$$
a_{i, 1}=\bar{p}_{1} a_{i+1}-a_{1} \bar{p}_{1}^{2} \cdots \bar{p}_{i}^{2} \bar{p}_{i+1}
$$

$$
\tau_{i, 1}=\tilde{y}_{1}^{\bar{a}_{0} a_{i+1}}
$$

a unit in $R_{1}$ for $i \geq 1$. Then we have expressions

$$
P_{i+1,1}=P_{i, 1}^{\bar{p}_{i+1}^{2}}-(1+t) \tau_{i, 1}^{\bar{p}_{i+1}} x_{1}^{\bar{p}_{i+1} a_{i, 1}}
$$

for $i \geq 1$. We have

$$
\nu\left(x_{1}\right)=\frac{1}{\bar{p}_{1}} \nu(x)=\frac{1}{\bar{p}_{1}} \text { and } \nu\left(P_{i, 1}\right)=\frac{a_{i, 1}}{\bar{p}_{i+1}} \nu\left(x_{1}\right)
$$

with $\operatorname{gcd}\left(a_{i, 1}, \bar{p}_{i+1}\right)=1$ for all $i \geq 1$.

We have that

$$
\begin{gathered}
R_{1} / m_{R_{1}}=R_{0} / m_{R_{0}}\left[\frac{P_{1}^{\bar{p}_{2}}}{P_{0}^{a_{1}}}\right]=R_{0} / m_{R_{0}}\left[\alpha_{1}\right]=k(t)\left(\alpha_{1}\right), \\
\bar{n}_{i, 1}:=\left[G\left(\nu\left(P_{0,1}\right), \ldots, \nu\left(P_{i, 1}\right)\right): G\left(\nu\left(P_{0,1}\right), \ldots, \nu\left(P_{i-1,1}\right)\right)\right]=\bar{n}_{i+1}=\bar{p}_{i+1}
\end{gathered}
$$

for $i \geq 1$ and setting $U_{i, 1}=x_{1}^{a_{i, 1}}$ for $i \geq 1$ and

$$
\alpha_{i, 1}=\left[\frac{P_{i, 1}^{\bar{p}_{i+1}}}{U_{i, 1}}\right] \in V_{\nu} / m_{\nu}
$$

Let $\bar{\tau}_{i, 1} \in R_{1} / m_{R_{1}}=k(t)\left(\alpha_{1}\right)$ be the class of $\tau_{i, 1}$ in $V_{\nu} / m_{\nu}$. We have that

$$
f_{i, 1}(u)=u^{\bar{p}_{i+1}}-(1+t) \bar{\tau}_{i, 1}^{\bar{p}_{i+1}}
$$

is the minimal polynomial of $\alpha_{i, 1}$ over

$$
\left.R_{1} / m_{R_{1}}\right)\left(\alpha_{1,1}, \ldots, \alpha_{i-1,1}\right)=k(t)\left(\alpha_{1}, \ldots, \alpha_{i}\right) .
$$

Thus

$$
d_{i, 1}:=\left[R_{1} / m_{R_{1}}\left(\alpha_{1,1}, \ldots, \alpha_{i, 1}\right): R_{1} / m_{R_{1}}\left(\alpha_{1,1}, \ldots, \alpha_{i-1,1}\right)\right]=\bar{p}_{i+1}
$$


and

for $i \geq 1$.

$$
n_{i, 1}:=d_{i, 1} \bar{n}_{i, 1}=\bar{p}_{i+1}^{2}
$$

Iterating this construction, we have an infinite sequence of birational extensions of regular local rings

$$
R_{0} \rightarrow R_{1} \rightarrow \cdots \rightarrow R_{j} \rightarrow \cdots
$$

which are dominated by $\nu$ where $R_{j}$ has regular parameters $x_{j}, y_{j}$ and a generating sequence $\left\{P_{i, j}\right\}$ for $\nu$ defined by

and for $i \geq 1$,

$$
P_{0, j}=x_{j}, P_{1, j}=y_{j}
$$

$$
P_{i+1, j}=P_{i, j}^{\bar{p}_{i+j}^{2}}-(1+t) \tau_{i, j}^{\bar{p}_{i+j}} x_{j}^{\bar{p}_{i+j} a_{i, j}}
$$

where $\tau_{i, j}$ are units in $R_{j}$ and $\operatorname{gcd}\left(a_{i, j}, \bar{p}_{i+j}\right)=1$. We have

$$
\nu\left(x_{j}\right)=\frac{1}{\bar{p}_{1} \cdots \bar{p}_{j}} \text { and } \nu\left(P_{i, j}\right)=\frac{a_{i, j}}{\bar{p}_{i+j}} \nu\left(x_{j}\right)
$$

for $i \geq 1$.

We have that

$$
\begin{gathered}
R_{j} / m_{R_{j}}=R_{j-1} / m_{R_{j-1}}\left[\frac{P_{1, j-1}^{\bar{p}_{1+j}}}{P_{0, j-1}^{a_{1, j-1}}}\right]=R_{j-1} / m_{R_{j-1}}\left[\alpha_{1, j-1}\right]=k(t)\left(\alpha_{1}, \ldots, \alpha_{j}\right), \\
\bar{n}_{i, j}:=\left[G\left(\nu\left(P_{0, j}\right), \ldots, \nu\left(P_{i, j}\right)\right): G\left(\nu\left(P_{0, j}\right), \ldots, \nu\left(P_{i-1, j}\right)\right)\right]=\bar{n}_{i+j}=\bar{p}_{i+j}
\end{gathered}
$$

for $i \geq 1$ and setting $U_{i, j}=x_{j}^{a_{i, j}}$ for $i \geq 1$ and

$$
\alpha_{i, j}=\left[\frac{P_{i, j}^{\bar{p}_{i+j}}}{U_{i, j}}\right] \in V_{\nu} / m_{\nu} .
$$

Let $\bar{\tau}_{i, j} \in R_{j} / m_{R_{j}}=k(t)\left(\alpha_{1}, \ldots, \alpha_{j}\right)$ be the class of $\tau_{i, j}$ in $V_{\nu} / m_{\nu}$. We have

$$
f_{i, j}(u)=u^{\bar{p}_{i+j}}-(1+t) \bar{\tau}_{i, j}^{\bar{p}_{i+j}}
$$

is the minimal polynomial of $\alpha_{i, j}$ over

$$
R_{j} / m_{R_{j}}\left(\alpha_{1, j}, \ldots, \alpha_{i-1, j}\right)=k(t)\left(\alpha_{1}, \ldots, \alpha_{i-1+j}\right) .
$$

Thus

and

$$
d_{i, j}:=\left[R / m_{R_{j}}\left(\alpha_{1, j}, \ldots, \alpha_{i, j}\right): R_{j} / m_{R_{j}}\left(\alpha_{1, j}, \ldots, \alpha_{i-1, j}\right)\right]=\bar{p}_{i+j}
$$

for $i \geq 1$.

$$
n_{i, j}:=d_{i, j} \bar{n}_{i, j}=\bar{p}_{i+j}^{2}
$$

Suppose $A$ is a regular local ring of the quotient field $K$ of $R_{0}$ which is dominated by $\nu$ and dominates $R_{0}$. Then there exists a largest $l$ such that $A$ dominates $R_{l}$, so there exist regular parameters $z$ and $w$ in $A$ such that $x_{l}$ and $y_{l}$ are monomials in $z$ and $w$. We thus have a factorization (by [2, Theorem 3])

$$
R_{l}=D_{0} \rightarrow D_{1} \rightarrow \cdots \rightarrow D_{n}=A
$$

where $D_{0}$ has regular parameters $z=x_{l}, w=y_{l}$ and $D_{i+1}$ has regular parameters $z_{i+1}$, $w_{i+1}$, such that either $z_{i}=z_{i+1}, w_{i}=z_{i+1} w_{i+1}$, or $z_{i}=z_{i+1} w_{i+1}, w_{i}=w_{i+1}$. 
We have shown that $D_{0}$ has a generating sequence $\left\{Q_{i}\right\}$ with $Q_{i}=P_{i . l}$ for $i \geq 0$, so that

$$
Q_{0}=z_{0}, Q_{1}=w_{0}
$$

and

$$
Q_{i+1}=Q_{i}^{\bar{p}_{i+l}^{2}}-(1+t) \tau_{i, l}^{\bar{p}_{i+l}} z_{0}^{\bar{p}_{i+l} e_{i, 0}}
$$

where $e_{i, 0}=a_{i, l}$ for $i \geq 1$. We have $D_{0} / m_{D_{0}}=k(t)\left(\alpha_{1}, \ldots, \alpha_{l}\right)$. Set

$$
\gamma_{i}=\alpha_{i, l}=\left[\frac{Q_{i}^{\bar{p}_{i+l}}}{Q_{0}^{e_{i, 1}}}\right]
$$

for $i \geq 1$. We have that

$$
f_{i, l}(u)=u^{\bar{p}_{i+l}}-(1+t) \overline{\bar{\tau}}_{i, l}^{\bar{p}_{i+l}}
$$

is the minimal polynomial of $\gamma_{i}$ over

$$
D_{0} / m_{D_{0}}\left(\gamma_{1}, \ldots, \gamma_{i-1}\right)=k(t)\left(\alpha_{1}, \ldots, \alpha_{i-1+l}\right)
$$

so

$$
\left[D_{0} / m_{D_{0}}\left(\gamma_{1}, \ldots, \gamma_{i}\right): D_{0} / m_{D_{0}}\left(\gamma_{1}, \ldots, \gamma_{i-1}\right)\right]=\bar{p}_{i+l}
$$

We will show by induction on $j$ that $D_{j}$ has a generating sequence $\left\{Q_{i, j}\right\}$ defined by

$$
\begin{gathered}
Q_{0, j}=z_{j}, Q_{1 j}=w_{j} \\
Q_{2, j}=Q_{1, j}^{\bar{p}_{1+l} c_{j}}-(1+t) \tau_{1, l}^{\bar{p}_{1+l}} Q_{0, j}^{\bar{p}_{1+l} e_{1, j}}
\end{gathered}
$$

with $c_{j}=\left[G\left(\nu\left(Q_{0, j}, \nu\left(Q_{1, j}\right)\right): G\left(\nu\left(Q_{1, j}\right)\right)\right]\right.$ and $\nu\left(Q_{1, j}^{c_{j}}\right)=\nu\left(Q_{0, j}^{e_{1, j}}\right)$ with $\operatorname{gcd}\left(c_{j}, e_{1, j}\right)=1$ and for $i \geq 2$,

$$
Q_{i+1, j}=Q_{i, j}^{\bar{p}_{i+l}^{2}}-(1+t) \tau_{i, l}^{\bar{p}_{i+l}} Q_{0, j}^{\bar{p}_{i+l} e_{i, j}} Q_{1, j}^{\bar{p}_{i+l} f_{i, j}}
$$

with $\nu\left(Q_{i, j}^{\bar{p}_{i+l}}\right)=\nu\left(Q_{0, j}^{e_{i, j}} Q_{1, j}^{f_{i, j}}\right)$, and with

$$
\bar{p}_{i+j}=\left[G\left(\nu\left(Q_{0, j}\right), \ldots, \nu\left(Q_{i, j}\right)\right): G\left(\nu\left(Q_{0, j}\right), \ldots, \nu\left(Q_{i-1, j}\right)\right)\right] .
$$

Further,

and

$$
\nu\left(Q_{2, j}\right)>\bar{p}_{1+l} c_{j} \nu\left(Q_{1, j}\right)=\bar{p}_{1+l} e_{1, j} \nu\left(Q_{0, j}\right)
$$

for $s>2$.

$$
\nu\left(Q_{s, j}\right)>\bar{p}_{s+l-1}^{2} \nu\left(Q_{s-1, j}\right)
$$

We also have that $D_{j} / m_{D_{j}}=k(t)\left(\alpha_{1}, \ldots, \alpha_{l}\right)$ and

$$
\gamma_{1}=\left[\frac{Q_{1, j}^{c_{j}}}{Q_{0, j}^{e_{1, j}}}\right] \text { and } \gamma_{i}=\left[\frac{Q_{i, j}^{\bar{p}_{i+l}}}{Q_{0, j}^{e_{i, j}} Q_{1, j}^{f_{i, j}}}\right] \in V_{\nu} / m_{\nu}
$$

for $i \geq 2$.

We inductively construct the generating sequence $Q_{0, j}, Q_{1, j}, \ldots$ as follows. Suppose that $Q_{0, j}, Q_{1, j}, \ldots$ has been constructed. We will construct $Q_{0, j+1}, Q_{1, j+1}, \ldots$. We either have that

$$
z_{j}=z_{j+1} w_{j+1}, w_{j}=w_{j+1}
$$

or

$$
z_{j}=z_{j+1}, w_{j}=z_{j+1} w_{j+1}
$$


Suppose (10) holds, so that $\nu\left(w_{j}\right)<\nu\left(z_{j}\right)$. Then

$$
w_{j+1}=Q_{1, j+1}=Q_{1, j} \text { and } z_{j+1}=Q_{0, j+1}=\frac{Q_{0, j}}{Q_{1, j+1}} .
$$

Substitute for $Q_{0, j}, Q_{1, j}$ in

$$
Q_{2, j}=Q_{1, j}^{\bar{p}_{1+l} c_{j}}-(1+t) \tau_{1, l}^{\bar{p}_{1+l}} Q_{0, j}^{e_{1, j} \bar{p}_{1+l}},
$$

to obtain

$$
Q_{2, j}=Q_{1, j+1}^{\bar{p}_{1+l} c_{j}}-(1+t) \tau_{1, l}^{\bar{p}_{1+l}} Q_{0, j+1}^{e_{1, j} \bar{p}_{1+l}} Q_{1, j+1}^{\bar{p}_{1+l} e_{1, j}} .
$$

Since $\nu\left(Q_{0, j}\right)>\nu\left(Q_{1, j}\right)$, and $\bar{p}_{1+l} c_{j} \nu\left(Q_{1, j}\right)=\bar{p}_{1+l} e_{1, j} \nu\left(Q_{0, j}\right)$, we have $\bar{p}_{1+l} e_{1, j}<\bar{p}_{1+l} c_{j}$. Thus a strict transform of $Q_{2, j}$ in $D_{j+1}$ is

$$
Q_{2, j+1}=\frac{Q_{2, j}}{Q_{1, j+1}^{\bar{p}_{1+l} e_{1, j}}}=Q_{1, j+1}^{\bar{p}_{1+l}\left(c_{j}-e_{1, j}\right)}-(1+t) \tau_{1, l}^{\bar{p}_{1+l}} Q_{0, j+1}^{\bar{p}_{1+l} e_{1, j}} .
$$

Suppose we have constructed the generating sequence out to

$$
Q_{i, j+1}=\frac{Q_{i, j}}{Q_{1, j+1}^{e_{1, j} \bar{p}_{1+l} \bar{p}_{l+2}^{2} \cdots \bar{p}_{l+i-1}^{2}}} .
$$

Substituting into

$$
Q_{i+1, j}=Q_{i, j}^{\bar{p}_{i+l}^{2}}-(1+t) \tau_{i, l}^{\bar{p}_{i+l}} Q_{0, j}^{\bar{p}_{i+l} e_{i, j}} Q_{1, j}^{\bar{p}_{i+l} f_{i, j}}
$$

we have

$$
Q_{i+1, j}=Q_{1, j+1}^{e_{1, j} \bar{p}_{1+l} \bar{p}_{l+2}^{2} \cdots \bar{p}_{l+i}^{2}} Q_{i, j+1}^{\bar{p}_{i+l}^{2}}-(1+t) \tau_{i, l}^{\bar{p}_{i+l}} Q_{0, j+1}^{\bar{p}_{i+l} e_{i, j}} Q_{1, j+1}^{\bar{p}_{i+l}\left(e_{i, j}+f_{i, j}\right)}
$$

We have that

$$
\bar{p}_{i+l}^{2} \nu\left(Q_{i, j}\right)=\bar{p}_{i+l} e_{i, j} \nu\left(Q_{0, j}\right)+\bar{p}_{i+l} f_{i, j} \nu\left(Q_{1, j}\right)<\bar{p}_{i+l}\left(e_{i, j}+f_{i, j}\right) \nu\left(Q_{0, j}\right)
$$

since $\nu\left(Q_{1, j}\right)<\nu\left(Q_{0, j}\right)$, and from the inequalities

$$
\nu\left(Q_{s, j}\right)>\bar{p}_{s+l-1}^{2} \nu\left(Q_{s-1, j}\right)
$$

for $s>2$ and

we have

$$
\nu\left(Q_{2, j}\right)>\bar{p}_{1+l} e_{1, j} \nu\left(Q_{0, j}\right)
$$

and thus

$$
e_{1, j} \bar{p}_{1+l} \bar{p}_{2+l}^{2} \cdots \bar{p}_{i+l}^{2}<\bar{p}_{i+l}\left(e_{i, j}+f_{i, j}\right)
$$

$Q_{i+1, j+1}=\frac{Q_{i+1, j}}{Q_{1, j+1}^{e_{1, j} \bar{p}_{1+l} \bar{p}_{2+l}^{2} \cdots \bar{p}_{i+l}^{2}}}=Q_{i, j+1}^{\bar{p}_{i+l}^{2}}-(1+t) \tau_{i, l}^{\bar{p}_{i+l}} Q_{0, j+1}^{\bar{p}_{i+l} e_{i, j}} Q_{1, j+1}^{\bar{p}_{i+l}\left(e_{i, j}+f_{i, j}-e_{1, j} \bar{p}_{1+l} \bar{p}_{2+l}^{2} \cdots \bar{p}_{i-1+l}^{2} \bar{p}_{i+l}\right)}$

is a strict transform of $Q_{i+1, j}$ in $D_{j+1}$.

Now suppose (11) holds, so that $\nu\left(w_{j}\right)>\nu\left(z_{j}\right)$. Then

$$
z_{j+1}=Q_{0, j+1}=Q_{0, j} \text { and } w_{j+1}=Q_{1, j+1}=\frac{Q_{1, j}}{Q_{0, j+1}} .
$$

Substitute for $Q_{0, j}$ and $Q_{1, j}$ in

$$
Q_{2, j}=Q_{1, j}^{\bar{p}_{1, l} c_{j}}-(1+t) \tau_{1, l}^{\bar{p}_{1+l}} Q_{0, j}^{\bar{p}_{1+l} e_{1, j}}
$$

to obtain

$$
Q_{2, j}=Q_{0, j+1}^{\bar{p}_{1+l} c_{j}} Q_{1, j+1}^{\bar{p}_{1+l} c_{j}}-(1+t) \tau_{8}^{\bar{p}_{1+l}{ }^{2}} Q_{0, j+1}^{\bar{p}_{1+l} e_{1, j}} .
$$


Since $\nu\left(Q_{1, j}\right)>\nu\left(Q_{0, j}\right)$ and $\bar{p}_{1+l} c_{j} \nu\left(Q_{1, j}\right)=\bar{p}_{1+l} e_{1, j} \nu\left(Q_{0, j}\right)<\bar{p}_{1+l} e_{1, j} \nu\left(Q_{1, j}\right)$ we have $\bar{p}_{1+l} c_{j}<\bar{p}_{1+l} e_{1, j}$. Thus a strict transform of $Q_{2, j}$ in $D_{j+1}$ is

$$
Q_{2, j+1}=\frac{Q_{2, j}}{Q_{0, j+1}^{c_{j} \bar{p}_{1+l}}}=Q_{1, j+1}^{\bar{p}_{1+l} c_{j}}-(1+t) \tau_{1, l}^{\bar{p}_{1+l}} Q_{0, j+1}^{\bar{p}_{1+l}\left(e_{1, j}-c_{j}\right)} .
$$

Now suppose we have constructed the generating sequence out to

$$
Q_{i, j+1}=\frac{Q_{i, j}}{Q_{0, j+1}^{c_{j} \bar{p}_{1+l} \bar{p}_{2+l}^{2} \cdots \bar{p}_{i-1+l}^{2}}} .
$$

Then substituting into

$$
Q_{i+1, j}=Q_{i, j}^{\bar{p}_{i+l}^{2}}-(1+t) \tau_{i, l}^{\bar{p}_{i+l}} Q_{0, j}^{\bar{p}_{i+l} e_{i, j}} Q_{1, j}^{\bar{p}_{i+l} f_{i, j}},
$$

we have that

$$
Q_{i+1, j}=Q_{i, j+1}^{\bar{p}_{i+l}^{2}} Q_{0, j+1}^{c_{j} \bar{p}_{1+l} \bar{p}_{2+l}^{2} \cdots \bar{p}_{i+l}^{2}}-(1+t) \tau_{i, l}^{\bar{p}_{i+l}} Q_{0, j+1}^{\bar{p}_{i+l}\left(e_{i, j}+f_{i, j}\right)} Q_{1, j+1}^{\bar{p}_{i+l} f_{i, j}} .
$$

We have

$$
\bar{p}_{i+l}^{2} \nu\left(Q_{i, j}\right)=\bar{p}_{i+l} e_{i, j} \nu\left(Q_{0, j}\right)+\bar{p}_{i+l} f_{i, j} \nu\left(Q_{1, j}\right)<\bar{p}_{i+l}\left(e_{i, j}+f_{i, j}\right) \nu\left(Q_{1, j}\right)
$$

since $\nu\left(Q_{0, j}\right)<\nu\left(Q_{1, j}\right)$, and from the inequalities

$$
\nu\left(Q_{s, j}\right)>\bar{p}_{s-1+l}^{2} \nu\left(Q_{s-1, j}\right)
$$

for $s>2$ and

we have

$$
\nu\left(Q_{2, j}\right)>\bar{p}_{1+l} c_{j} \nu\left(Q_{1, j}\right)
$$

and thus

$$
\bar{p}_{i+l}^{2} \bar{p}_{i-1+l}^{2} \cdots \bar{p}_{2+l}^{2} \bar{p}_{1+l} c_{j}<\bar{p}_{i+l}\left(e_{i, j}+f_{i, j}\right)
$$

$$
Q_{i+1, j+1}=\frac{Q_{i+1, j}}{Q_{0, j+1}^{c_{j} \bar{p}_{1+l} \bar{p}_{2+l}^{2} \cdots \bar{p}_{i+l}^{2}}}=Q_{i, j+1}^{\bar{p}_{i+l}^{2}}-(1+t) \tau_{i, l}^{\bar{p}_{i+l}} Q_{0, j+1}^{\bar{p}_{i+l}\left(e_{i, j}+f_{i, j}-c_{j} \bar{p}_{1+l} \bar{p}_{2+l}^{2} \cdots \bar{p}_{i-1+l}^{2} \bar{p}_{i+l}\right)} Q_{1, j+1}^{\bar{p}_{i+l} f_{i, j}}
$$

is a strict transform of $Q_{i+1, j}$ in $D_{j+1}$.

Since $\operatorname{gcd}\left(c_{j}, e_{1, j}\right)=1$,

$$
\left[G\left(\nu\left(Q_{0, j}\right), \nu\left(Q_{1, j}\right)\right): G\left(\nu\left(Q_{0, j}\right)\right)\right]=c_{j}
$$

and since $G\left(\nu\left(Q_{0, j+1}\right), \nu\left(Q_{1, j+1}\right), \ldots, \nu\left(Q_{s, j+1}\right)\right)=G\left(\nu\left(Q_{0, j}\right), \nu\left(Q_{1, j}\right), \ldots, \nu\left(Q_{s, j}\right)\right)$ for $s \geq$ 1 , we have

$$
\left[G\left(\nu\left(Q_{0, j}\right), \nu\left(Q_{1, j}\right), \ldots, \nu\left(Q_{i, j}\right)\right): G\left(\nu\left(Q_{0, j}\right), \nu\left(Q_{1, j}\right), \ldots, \nu\left(Q_{i-1, j}\right)\right)\right]=\bar{p}_{i+l}
$$

for $i \geq 2$. Dividing the relation (7) by $Q_{0, j}^{\bar{p}_{1+l} e_{1, j}}$ and taking the residue in $V_{\nu} / m_{\nu}$, we obtain

$$
0=\left[\frac{Q_{2, j}}{Q_{0, j}^{\bar{p}_{1+l} e_{1, j}}}\right]=f_{1, l}\left(\gamma_{1}\right)
$$

where $f_{1, l}$, defined by (5), is the minimal polynomial of $\gamma_{1}$ over $D_{j} / m_{D_{j}}=k(t)\left(\alpha_{1}, \ldots, \alpha_{l}\right)$. Dividing the relation (8) by $\left(Q_{0, j}^{e_{i, j}} Q_{1, j}^{f_{i, j}}\right)^{\bar{p}_{i+l}}$, and taking the residue in $V_{\nu} / m_{\nu}$, we obtain

$$
0=\left[\frac{Q_{i+1, j}}{\left(Q_{0, j}^{e_{i, j}} Q_{1, j}^{f_{i, j}}\right)^{\bar{p}_{i+l}}}\right]=f_{i, l}\left(\gamma_{i}\right),
$$


where $f_{i, l}$, defined by (5), is the minimal polynomial of $\gamma_{i}$ over $D_{j} / m_{D_{j}}\left(\gamma_{1}, \ldots, \gamma_{i-1}\right)$ for $i \geq 2$.

We now verify (9) by induction on $j$. If we are in case (10), then the formula follows for $j+1$ from induction and (12), (13) and (14). If we are in case (11), then the formula follows for $j+1$ from induction and (15), (16) and (17).

The formula $D(i)$ of [ 8 , Theorem 4.2] holds for the $Q_{0, j}, Q_{1, j}, \ldots$ That is, if we have a natural number $i$, a positive integer $m$ and natural numbers $f_{n}(s)$ for $1 \leq n \leq i$ such that $0 \leq f_{1}(s)<\bar{p}_{1+l} c_{j}$ for $1 \leq s \leq m$ and $0 \leq f_{n}(s)<\bar{p}_{n+l}^{2}$ for $2 \leq s \leq m$ and $1 \leq n \leq i$. If

$$
\nu\left(Q_{0, j}^{f_{0}(s)} Q_{1, j}^{f_{1}(s)} \cdots Q_{i, j}^{f_{i}(s)}\right)=\nu\left(Q_{0, j}^{f_{0}(1)} Q_{1, j}^{f_{1}(1)} \cdots Q_{i, j}^{f_{i}(1)}\right)
$$

for $1 \leq s \leq m$, then

$$
1,\left[\frac{Q_{0 . j}^{f_{0}(2)} Q_{1, j}^{f_{1}(2)} \cdots Q_{i, j}^{f_{i}(2)}}{Q_{0 . j}^{f_{0}(1)} Q_{1, j}^{f_{1}(1)} \cdots Q_{i, j}^{f_{i}(1)}}\right], \cdots,\left[\frac{Q_{0 . j}^{f_{0}(m)} Q_{1, j}^{f_{1}(m)} \cdots Q_{i, j}^{f_{i}(m)}}{Q_{0 . j}^{f_{0}(1)} Q_{1, j}^{f_{1}(1)} \cdots Q_{i, j}^{f_{i}(1)}}\right]
$$

are linearly independent over $D_{j} / m_{D_{j}}=k(t)\left(\alpha_{1}, \ldots, \alpha_{l}\right)$. This formula follows from induction on $i$ and (9), (5), (18) and (19) as in the proof of $D(i)$ of [8, Theorem 4.2]. Since $\nu$ has rank 1 by (2), the fact that $Q_{0, j}, Q_{1, j}, \ldots$ is a generating sequence in $D_{j}$ is verified as in the proof of [ 8 , Theorem 4.10 and Lemma 4.9].

\section{Construction of unramified extensions Which have Larger Valuation SEMIGROUPS}

Let notation be as in the previous section. Let $K=k(t, x, y)$. Let $A$ be a regular local ring of $K$ which dominates $R_{0}$ and is dominated by $\nu$. Then there exists a factorization of

$$
R_{0} \rightarrow R_{l}=D_{0} \rightarrow D_{n}=A
$$

of the form (41). Let

$$
\lambda \text { be a } \bar{p}_{1+l^{-}} \text {th root of } 1+t
$$

in an algebraic closure of $K, L=K(\lambda)$ and $\bar{\nu}$ be an extension of $\nu$ to $L$. Let $\omega \in k$ be a primitive $\bar{p}_{1+l}$-th root of unity.

Let $\bar{p}=\bar{p}_{1+l}$ and $f(u)=u^{\bar{p}}-(1+t)$, the minimal polynomial of $\lambda$ over $K$. Let $B=A[\lambda]$. The ring $B$ is finite over $A$. We have the formula for the discriminant

$$
D_{L / K}\left(1, \lambda, \ldots, \lambda^{\bar{p}-1}\right)=(-1)^{\frac{\bar{p}(\bar{p}-1)}{2}} \prod_{i=1}^{\bar{p}} \frac{d f}{d u}\left(\omega^{i} \lambda\right)
$$

by [13, Proposition 8.5 on page 204] and [13, Formula (4) on page 204]. Thus

$$
D_{L / K}\left(1, \lambda, \ldots, \lambda^{\bar{p}-1}\right)=(-1)^{\frac{\bar{p}(\bar{p}-1)}{2}} \bar{p}^{\bar{p}}(1+t)^{\bar{p}-1} .
$$

Now $1+t \in R_{0}$ and $1+t \notin m_{R_{0}}$, so $1+t \notin m_{A}$. Thus the discriminant ideal $D(B / A)$ in $A$ is equal to $A$, and so $B$ is normal and $A \rightarrow B$ is unramified by [3, Proposition 1.43] and [3. Theorem 1.44]. Let $C=B_{m_{\bar{\nu}} \cap B}$. Then $A \rightarrow C$ is unramified, and so $C$ is a regular local ring, since the maximal ideal of $C$ is generated by a regular system of parameters in A.

Proposition 4.1. With the above notation, we have inequalities of semigroups

$$
S^{C}(\bar{\nu}) \neq S^{A}(\nu)
$$

under the natural inclusion $S^{A}(\nu) \subset S^{C}(\bar{\nu})$. 
Proof. Since $A=D_{n}$ in (4), $A$ has regular parameters $z_{n}, w_{n}$ and a generating sequence

$$
Q_{0, n}=z_{n}, Q_{1, n}=w_{n}, Q_{2, n}, \ldots
$$

defined by (6), (7) and (마). so

$$
S^{A}(\nu)=S\left(\left\{\nu\left(Q_{i, n}\right) \mid i \geq 0\right\}\right) .
$$

We have

$$
\nu\left(Q_{i+1, n}\right)>\nu\left(Q_{i, n}\right)
$$

for $i \geq 1$,

$$
\left[G\left(\nu\left(Q_{0,1}\right), \nu\left(Q_{1, n}\right)\right): G\left(\nu\left(Q_{0, n}\right)\right]=c_{n}\right.
$$

and

$$
\left[G\left(\nu\left(Q_{0, n}\right), \nu\left(Q_{1, n}\right), \ldots, \nu\left(Q_{i+1, n}\right)\right): G\left(\nu\left(Q_{0, n}\right), \nu\left(Q_{1, n}\right), \ldots, \nu\left(Q_{i, n}\right)\right)\right]=\bar{p}_{i+l+1}
$$

for $i \geq 1$.

By (7), we have

$$
Q_{2, n}=Q_{1, n}^{\bar{p}_{1+l} c_{n}}-(1+t) \tau_{1, l}^{\bar{p}_{1+l}} Q_{0,1}^{\bar{p}_{1+l} e_{1, n}}=w_{n}^{\bar{p}_{1+l} c_{n}}-(1+t) \tau_{1, l}^{\bar{p}_{1+l}} z_{n}^{\bar{p}_{1+l} e_{1, n}} .
$$

We have

$$
\gamma_{1}=\left[\frac{w_{n}^{c_{n}}}{z_{n}^{e_{1, n}}}\right] \in V_{\nu} / m_{\nu} \subset V_{\bar{\nu}} / m_{\bar{\nu}} .
$$

Let $\beta=\left[\lambda \tau_{i, l}\right] \in V_{\bar{\nu}} / m_{\bar{\nu}}$ which is nonzero since $\lambda$ and $\tau_{1, l}$ are units in $C$. There exists at most one index $j$ with $1 \leq j \leq \bar{p}_{1+l}$ such that $\omega^{j} \beta=\gamma_{1}$. We have that

$$
h_{j}=w_{n}^{\bar{p}_{1+l}}-\omega^{j} \lambda \tau_{1, j} z_{n}^{e_{1, n}} \in C
$$

for all $j$. If $\omega^{j} \beta \neq \gamma_{1}$, then $\bar{\nu}\left(h_{j}\right)=e_{1, n} \nu\left(z_{n}\right)$. Since

$$
\sum_{j=1}^{\bar{p}_{1+l}} \bar{\nu}\left(h_{j}\right)=\nu\left(Q_{2, n}\right)>\bar{p}_{1+l} e_{1, n} \nu\left(z_{n}\right),
$$

there exists a unique value of $j$ such that $\omega^{j} \beta=\gamma_{1}$, and $\bar{\nu}\left(h_{j}\right)>e_{1, n} \nu\left(z_{n}\right)$. If $\bar{\nu}\left(h_{j}\right) \in$ $S^{A}(\nu)$, we must then have that $\bar{\nu}\left(h_{j}\right) \in S\left(\nu\left(z_{n}\right), \nu\left(w_{n}\right)\right)$, since

$$
\bar{\nu}\left(h_{j}\right)=\nu\left(Q_{2, n}\right)-\left(\bar{p}_{1+l}-1\right) e_{1, n} \nu\left(z_{n}\right)<\nu\left(Q_{2, n}\right)
$$

and by (23). Thus $\nu\left(Q_{2, n}\right) \in G\left(\nu\left(z_{n}\right), \nu\left(w_{n}\right)\right)$, which is a contradiction to (24).

Let $\mu$ be a valuation of $V_{\nu} / m_{\nu}=k(t)\left[\left\{(1+t)^{\frac{1}{\bar{p}_{i}}}\right\}_{i \in \mathbb{Z}_{+}}\right]$which is an extension of the $(t)$-adic valuation on $k[t]_{(t)}$. The value group of $\mu$ is $\mathbb{Z}$. Let $\varphi$ be the composite valuation of $\nu$ and $\mu$ on $K$, so that the valuation ring of $\varphi$ is $V_{\varphi}=\pi^{-1}\left(V_{\mu}\right)$ where $\pi: V_{\nu} \rightarrow V_{\nu} / m_{\nu}$ is the residue map ([3, Section 10]). The residue field of $\varphi$ is $V_{\varphi} / m_{\varphi}=V_{\mu} / m_{\mu} \cong k$. Let $T_{0}=k[t, x, y]_{(t, x, y)}$, which is dominated by $\varphi$.

Proposition 4.2. Suppose that $T$ is a regular local ring of $K$ which dominates $T_{0}$ and is dominated by $\varphi$. Then there exists a finite separable extension field $L$ of $K$ such that $T$ is unramified in $L$. Further, if $\bar{\varphi}$ is an extension of $\varphi$ to $L$, and if $U$ is the normal local ring of $L$ which lies over $T$ and is dominated by $\bar{\varphi}$, then the following properties hold:

1) $U$ is a regular local ring

2) the extension $T \rightarrow U$ is unramified with no residue field extension

3) $S^{U}(\bar{\varphi}) \neq S^{T}(\varphi)$ under the natural inclusion $S^{T}(\varphi) \subset S^{U}(\bar{\varphi})$. 
Proof. Let $R_{0}=\left(T_{0}\right)_{m_{\nu} \cap T_{0}}=k(t)[x, y]$ and $A=T_{m_{\nu} \cap T}$. By consideration of the factorization $R_{0} \rightarrow R_{l} \rightarrow A=D_{n}$ in (4), let $\lambda$ be a $\bar{p}_{1+l^{-}}$th root of $1+t$ in an extension field of $K$, and let $L=K(\lambda)$ (as in (22) ). Let $\bar{\varphi}$ be an extension of $\varphi$ to $L$ and let $\bar{\nu}$ be the extension of $\nu$ to $L$ with which $\bar{\varphi}$ is composite.

Let $U$ be the normal local ring of $L$ which is dominated by $\bar{\varphi}$ and lies over $T$. Then $U$ is a regular local ring and the extension $T \rightarrow U$ is unramified with no residue field extension, by the argument before Proposition 4.1 and since $V_{\bar{\varphi}} / m_{\bar{\varphi}}=V_{\varphi} / m_{\varphi}=k$ by [21, Corollary 2, page 26]. Let $C=U_{m_{\bar{\nu}} \cap U}$. Then $C$ is a regular local ring and $A \rightarrow C$ is unramified (by the argument before Proposition 4.1). We have that

$$
S^{C}(\bar{\nu}) \neq S^{A}(\nu)
$$

by Proposition 4.1

By the explanation on [3, page 56] or [21, Theorem 17, page 43], we have a commutative diagram of homomorphisms of value groups, where the horizontal sequences are short exact and the vertical arrows are injective,

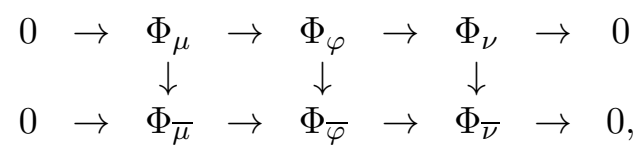

which induce a commutative diagram of homorphisms of semigroups, where the horizontal arrows are surjective and the vertical are injective,

$$
\begin{array}{ccc}
S^{T}(\varphi) & \rightarrow & S^{A}(\nu) \\
\downarrow & & \downarrow \\
S^{U}(\bar{\varphi}) & \rightarrow & S^{C}(\bar{\nu}) .
\end{array}
$$

We will show that the first horizontal arrow is surjective. The proof for the second horizontal arrow is the same. Suppose $\alpha \in S^{A}(\nu)$. Then there exists $f \in A$ such that $\nu(f)=\alpha$. There exists $g \in T \backslash(x, y)$ such that $g f \in T$. Thus $\varphi(g) \in \Phi_{\mu}$ and $\varphi(g f)=\varphi(g)+\varphi(f)$ so $\varphi(g f) \in S^{T}(\varphi)$ maps onto $\nu(f)=\alpha$. Thus $S^{T}(\varphi) \neq S^{U}(\bar{\varphi})$ by (25) .

\section{Proofs of Theorems 1.5 and 1.6}

We first give the proof of Theorem 1.5 .

A Henselization $T^{h}$ of $T$ can be constructed as follows, as is explained in [15, Chapter VII]. Let $N$ be a separable closure of $K$. Then $N$ is an (infinite) Galois extension of $K$ with Galois group $G(N / K)$. Let $E$ be a local ring of the integral closure of $T$ in $N$, and let

$$
G^{s}(E / T)=\{\sigma \in G(N / K) \mid \sigma(E)=E\} .
$$

A Henselization $T^{h}$ of $T$ is then $T^{h}=E^{G^{s}(E / T)}$, which is a local ring of the fixed field $M=N^{G^{s}(E / T)}$ of $G^{s}(E / T)$ which lies over $T$.

Let $K \rightarrow L$ be the field extension of Proposition 4.2. Choose an embedding $K \rightarrow L \rightarrow N$ of $L$ as a subfield of $N$, and let $U$ be the local ring of the integral closure of $T$ in $L$ which is dominated by $E$. By Proposition 4.2, $U$ is unramified over $T$ with no residue field extension. Thus $G^{s}(E / T) \subset G(N / L)$ (c.f. [2, Section 2] or [5, Section 4]). Thus we have that

$$
L=N^{G(N / L)} \subset N^{G^{s}(E / T)}=M .
$$

Thus $U$ is dominated by $T^{h}$ since $U=E \cap L$ and $T^{h}=E \cap M$. Let $\bar{\varphi}=\varphi^{h} \mid L$. Then $\bar{\varphi}$ dominates $U$ and $T^{h}$ dominates $U$, so $S^{U}(\bar{\varphi}) \subset S^{T^{h}}\left(\varphi^{h}\right)$. But $S^{U}(\bar{\varphi}) \neq S^{T}(\varphi)$ by Proposition 4.2, so $S^{T^{h}}\left(\varphi^{h}\right) \neq S^{T}(\varphi)$. 
We now give the proof of Theorem 1.6.

By [15, Theorem 43.5], the completion $\hat{T}$ of $T$ is a Henselian local ring, and so by [15, Theorem 30.3], $\hat{T}$ dominates the Henselization $T^{h}$ of $T$.

Now $T^{h}=F_{m}$ where $F$ is the integral closure of $T$ in the quotient field $M$ of $T^{h}$ and $m$ is a maximal ideal of $F$. Suppose $q$ is a nonzero prime ideal of $T^{h}$. Then there exists a nonzero element $f \in q$, so that $f=\frac{g}{h}$ where $g, h \in F$ and $h \notin m$. Now $g$ is integral over $T$ and $T$ is normal, so the norm $N_{K(g) / K}(g)$ satisfies $0 \neq N_{K(g) / K}(g) \in q \cap T$ by [20, Theorem 4, page 260] and [20, formula (15) on page 91]. Thus $q \cap T \neq(0)$.

Suppose there exists a prime ideal $p$ in $\hat{T}$ with an extension $\hat{\varphi}$ of $\varphi$ to the quotient field of $\hat{T} / p$ which dominates $\hat{T} / p$ such that $S^{\hat{T} / p}(\hat{\varphi})=S^{T}(\varphi)$. Then $p \cap T^{h}=(0)$, and so $\hat{T} / p$ dominates $T^{h}$. Let $\varphi^{h}$ be the restriction of $\hat{\varphi}$ to the quotient field of $T^{h}$. We then have natural inclusions

$$
S^{T}(\varphi) \subset S^{T^{h}}\left(\varphi^{h}\right) \subset S^{\hat{T} / p}(\hat{\varphi}) .
$$

But $S^{T}(\varphi) \neq S^{T^{h}}\left(\varphi^{h}\right)$ by Theorem [1.5, giving a contradiction to our assumption that $S^{\hat{T} / p}(\hat{\varphi})=S^{T}(\varphi)$.

\section{REFERENCES}

[1] S. Abhyankar, On the valuations centered in a local domain, Amer. J. Math. 78 (1956), 321 $-348$.

[2] S. Abhyankar, Local uniformization on algebraic surfaces over ground fields of characteristic $p \neq 0$, Annals Math. 63 (1956), 491 - 526.

[3] S. Abhyankar, Ramification theoretic methods in algebraic geometry, Princeton Univ. Press, 1959.

[4] S.D. Cutkosky, Local factorization and monomialization of morphisms, Astérisque 260, 1999.

[5] S.D. Cutkosky, Finite generation of extensions of associated graded rings along a valuation, to appear in the Journal of the London Math. Soc.

[6] S.D. Cutkosky and S. El Hitti, Formal prime ideals of infinite value and their algebraic resolution, Ann. Fact. Sci. Toulouse Math. 19 (2010), 635 - 649.

[7] S.D. Cutkosky and L. Ghezzi, Completions of valuation rings, Contemp. Math. 386 (2005), $13-34$.

[8] S.D. Cutkosky and Pham An Vinh, Valuation semigroups of two dimensional local rings, Proceedings of the London Mathematical Society 108 (2014), 350 - 384.

[9] S. El Hitti, Perron transforms, Comm. Algebra 42 (2014), 2004 - 2045.

[10] W. Heinzer and J. Sally, Extensions of valuations to the completion of a local domain, J. Pure and Appl. Algebra 71 (1991), 175 - 185.

[11] F.J. Herrera Govantes, F.J. Olalla Acosta, M. Spivakovsky, G. Teissier, Extending a valuation centered in a local domain to its formal completion, Proc. London Math. Soc. 105 (2012), $571-621$.

[12] O. Kashcheyeva, Constructing examples of semigroups of valuations, J. Pure Appl. Algebra 200 (2016), $3826-3860$.

[13] S. Lang, Algebra, Revised third edition, Springer Verlag, 2002

[14] M. Moghaddam, A construction for a class of valuations of the field $K\left(X_{1}, \ldots, X_{d}, Y\right)$ with large value group, Journal of Algebra, 319, 7 (2008), 2803-2829.

[15] M. Nagata, Local Rings, Interscience publishers, New York, London, 1962.

[16] J. Novacoski and M. Spivakovsky, Key polynomials and pseudo-convergent sequences, J. Algebra 495 (2018), 199 - 219.

[17] M. Spivakovsky, Valuations in function fields of surfaces, Amer. J. Math. 112 (1990), 107 156.

[18] B. Teissier, Valuations, deformations and toric geometry, Valuation theory and its applications II, F.V. Kuhlmann, S. Kuhlmann and M. Marshall, editors, Fields Institute Communications 33 (2003), Amer. Math. Soc., Providence, RI, 361 - 459. 
[19] B. Teissier, Overweight deformations of affine toric varieties and local uniformization, in Valuation theory in interaction, Proceedings of the second international conference on valuation theory, Segovia-El Escorial, 2011. Edited by A. Campillo, F-V- Kehlmann and B. Teissier. European Math. Soc. Publishing House, Congress Reports Series, Sept. 2014, 474 - 565.

[20] O. Zariski and P. Samuel, Commutative Algebra Volume I, Van Nostrand, 1958.

[21] O. Zariski and P. Samuel, Commutative Algebra Volume II, Van Nostrand, 1960.

Steven Dale Cutkosky, Department of Mathematics, University of Missouri, Columbia, MO 65211, USA

E-mail address: cutkoskys@missouri.edu 